\title{
Beta Lactamase Producing Escherichia coli, Klebsiella pneumoniae and Methicillin Resistant Staphylococcus aureus among Uropathogens
}

\author{
V.K.Singh ${ }^{l}$, R. Tuladhar ${ }^{2}$ and M.K.Chaudhary ${ }^{3}$ \\ ${ }^{1}$ Nepal Academy of Science and Technology, Lalitpur, Nepal \\ ${ }^{2}$ Central Department of Microbiology, Tribhuvan University, Nepal \\ ${ }^{3}$ Department of Microbiology, KIST MCTH, Lalitpur, Nepal \\ e-mail: viveksingh1424@gmail.com
}

\begin{abstract}
Urinary tract infection (UTI) is the leading cause of morbidity and health care expenditures in persons of all the ages. The impact of disease is high in the low-resource developing countries due to lack of awareness about the UTIs caused by extended spectrum beta lactamase producing pathogens and methicillin resistant Staphylococcus aureus. This study represents a cross-sectional analysis of UTI prevalence in patients attending at KIST Medical College Teaching Hospital, Imadole, Lalitpur. Bacterial isolates were identified using standard biochemical tests. The antimicrobial susceptibility to various drugs and confirmation of the extended spectrum B-lactamase (ESBL) and methicillin resistant Staphylococcus aureus (MRSA) production was done following Clinical \& Laboratory Standards Institute (CLSI) guidelines. During the study period, 303 (24.08\% of urine submissions) UTIs were confirmed. Among these 303 strains, E. coli was the most common, representing $65.34 \%$ of the isolates, followed by S. aureus $(6.93 \%)$ and other pathogens (27.72\%). Prevalence of ESBL E. coli, K. pneumoniae and MRSA in the urinary isolates were found to be $38.38 \%, 35 \%$ and $47.61 \%$ respectively. Empiric treatment for UTIs in Nepal should be reconsidered and prudent use of antibiotics, particularly Gentamycin, is highly recommended. The recent spread of ESBL-producing Enterobacteriaceae and MRSA is striking and underlines the need for further studies.
\end{abstract}

Key words: antimicrobial susceptibility pattern, ESBL, MDR, MRSA, Urinary tract infection

\section{Introduction}

The term urinary tract infection (UTI) refers to the invasion of the urinary tract by a non-resident infectious organisms. UTI encompasses a wide variety of clinical entities whose common denominator is microbial invasion of any tissue of the tract from the renal cortex to the urethral meatus (Pokhrel 2004). Bacteriuria which may lead to the infection of the prostate, epididymis or the testes are also included in the definition of UTI (Fowler \& Mariano 1990). UTI is among the most common reasons patients seek medical care. It is estimated that approximately $10 \%$ of humans will have UTI at some time during their lives (Boscia \& Kaye 1987).

Bacteria of only a limited number of species are able to initiate infection in the urinary tract. The causative agents are listed below (Cheesbrough 2010):
Gram negative E. coli, Proteus mirabilis, P. vulgaris, Klebsiella spp., Enterobacter spp., Citrobacter spp, Serratia spp., M. morganii and $P$. aeruginosa

Gram positive $\quad$ S. aureus, S. saprophyticus, Group B streptococci and E. faecalis

Other pathogens Chlamydia (Chlamydia trachomatis), Mycoplasma, Ureaplasma urealyticum, Candida spp. and Mycobacterium tuberculosis

Extended spectrum beta lactamases (ESBLs) are a group of enzymes with the ability to hydrolyze and cause resistance to the oxymino-cephalosporins (i.e. cefotaxime, ceftazidime, ceftriaxone, cefuroxime and cefepime) and monobactams (i.e. aztreonam) (Peirano \& Pitout 2010). Increasing resistance to thirdgeneration cephalosporins amongst $E$. coli and 
Klebsiella spp. is predominantly due to the production of extended-spectrum beta lactamases (ESBLs). ESBL producers are associated with increased morbidity and mortality, especially amongst patients on intensive care and high-dependency units. Accurate laboratory detection is important to avoid clinical failure due to inappropriate antimicrobial therapy.

Methicillin-resistant Staphylococcus aureus is a bacterium responsible for difficult-to-treat infections in humans. It may also be referred to as multidrugresistant Staphylococcus aureus or oxacillin-resistant Staphylococcus aureus. MRSA is by definition a strain of Staphylococcus aureus that is resistant to a large group of antibiotics called the beta-lactams, which include the penicillins and the cephalosporins. MRSA has evolved an ability to survive treatment with betalactam antibiotics, including methicillin, dicloxacillin, nafcillin, and oxacillin (Maucky 2011). This group of organisms has since emerged as a serious concern in human medicine (Barrettetal. 1968, Lee 2003, Pinhoet al. 2001, Weese et al. 2005).

\section{Methodology Specimen collection}

The patient was given a sterile, dry, wide-necked leakproof Hi-Media container of $100 \mathrm{ml}$ capacity for collection of 10-20 ml of clean-catch mid-stream urine. The patient was given instructions for the collection of CC-MSU.

\section{Culture of specimen}

The urine sample was cultured onto the MacConkey agar and Blood agar medium by the semi-quantitative culture technique using a standard loop (Sapkota et al. 2014).

\section{Identification of the isolates}

Identification of significant isolates was done by using microbiological techniques which involves morphological appearance of the colonies, staining reactions and biochemical properties (Cheesbrough1984, Collee et al. 1999, Forbes et al. 2002).

\section{Antibiotic susceptibility testing}

The antibiotic sensitivity testing was performed according to the recommended Kirby-Bauer sensitivity testing method (CLSI 2013).

\section{Quality control for test}

Quality of each test was maintained by using standard procedures. The quality of each agar plates prepared was tested by incubating one plate of each lot on the incubator. During identification of organism, for each test ATCC control positives and control negatives was taken simultaneously. Quality of sensitivity tests was maintained by maintaining the thickness of MuellerHinton agar at $4 \mathrm{~mm}$ and the $\mathrm{pH}$ at 7.2-7.4. Similarly antibiotic discs containing the correct amount as indicated were used. Strict aseptic conditions were maintained while carrying out all these procedures.

\section{Screening test for ESBLs}

Isolates were screened for ESBL production by using disc diffusion of cefotaxime (CTX), ceftazidime (CAZ), ceftriaxone (CTR) and Aztreonam (AT) placed on inoculated plates containing Muller Hinton agar according to the CLSI recommendations.

\section{Confirmatory test for ESBLs}

Phenotypic confirmatory test for ESBL was done by combination disc method for all the ESBL producing isolates as per CLSI 2013 guidelines.

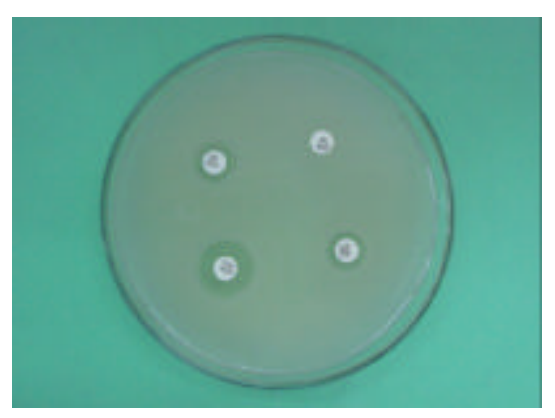

Fig.1. Phenotypic confirmatory test for ESBL E. coli $(\mathrm{CAZ}=$ Ceftazidime, $\mathrm{CAC}=$ Ceftazidime + Clavulanic acid, CTX $=$ Cefotaxime, $\mathrm{CEC}=$ Cefotaxime+Clavulanic acid

In this test a disc of ceftazidime $(30 \mu \mathrm{g})$, cefotaxime $(30 \mu \mathrm{g})$ alone and a disc of ceftazidime and cefotaxime in combination with clavulanic acid $(30 / 10 \mu \mathrm{g})$ were used for each isolates. Both the discs were placed 25 $\mathrm{mm}$ apart, centre to center, on a lawn culture of the test isolate on Muller Hinton agar plate and incubated overnight at $37^{\circ} \mathrm{C}$. A $\geq 5 \mathrm{~mm}$ increase in zone diameter for either antimicrobial agent tested in combination with clavulinic acid versus its zone when tested alone was designated as ESBL positive. 
Interpretation: An increase in inhibition zone diameter of $\geq 5 \mathrm{~mm}$ around combination disk of Ceftazidime + Clavulanic acid versus the inhibition zone diameter around Ceftazidime disk alone, confirms ESBL production.

\section{Identification of the MRSA}

Identification of $S$. aureus was done based on colony morphology on 5\% sheep blood agar, gram stain appearance and catalase test.

Detection of MRSA was done using cefoxitin disc. Bacterial colonies was suspended in saline to a turbidity of $0.5 \mathrm{McFarland}$ standards and inoculated on a Muller Hinton agar plate. Plates were incubated at $35^{\circ} \mathrm{C}$. All isolates resistant to cefoxitin were considered as MRSA (Mshana et al.2009).

\section{Statistical analysis}

Statistical package for social sciences (SPSS) version 16 was used to analyze the data.

\section{Results and Discussion}

Of the1258 specimens examined in this study, 303 (24.08\%) showed significant bacteriuria. Of the 303 bacterial isolates, the gram positive and negative bacteria accounted $15 \%$ and $83 . \%$ respectively. The rest were yeast $(0.99 \%)$. The gram negative bacteria included: Escherichia coli 198, Klebsiella pneumoniae 20, Klebsiella oxitoca 1, Proteus mirabilis 6, Proteus vulgaris 4, Citrobacter koseri 3, Citrobacter freundii 2, Enterobacter species 1, Pseudomonas aeruginosa 4, Acinetobacter species 14 and the gram positive were Staphylococus aureus 21, Coagulase negative Staphylococcus aureus 10, Streptococcus species 7, Enterococcus species 9 and the yeast included Candida albicans1, Non albicans Candida species 2.

The in-vitro antibiotic sensitivity pattern of the isolates to common anti-microbial agents is shown in Table 1 and 2. Results indicated that gentamycin and nitrofurantoin were the most potent of all the antibiotics. Norfloxacin, ampicillin, ciprofloxacin, ceftriaxone and cotri-moxazole were poorly effective.

Table 1. Antibiotic susceptibility pattern of gram positive bacteria isolated from urine samples.

\begin{tabular}{l|c|c|c|c}
\hline Antibiotic used & Sensitive & Intermediate & Resistant & Total \\
\hline Ampicillin & 25 & & 22 & 47 \\
Gentamicin & 32 & & 15 & 47 \\
Norfloxacin & 21 & & 26 & 47 \\
Nitrofurantoin & 38 & 2 & 7 & 47 \\
Ciprofloxacin & 23 & 1 & 23 & 47 \\
Cotrimoxazole & 24 & & 23 & 47 \\
Ceftriaxone & 24 & & 23 & 47 \\
\hline
\end{tabular}

Table 2. Antibiotic sensitivity pattern against gram negative bacteria isolated from urine samples.

\begin{tabular}{l|c|c|c|c}
\hline Antibiotic used & Sensitive & Intermediate & Resistant & Total \\
\hline Ampicillin & 50 & 2 & 201 & 253 \\
Gentamicin & 192 & 3 & 58 & 253 \\
Nalidixic acid & 62 & 3 & 188 & 253 \\
Norfloxacin & 111 & 3 & 139 & 253 \\
Nitrofurantoin & 148 & 39 & 66 & 253 \\
Ciprofloxacin & 123 & 1 & 129 & 253 \\
Cotrimoxazole & 107 & 1 & 145 & 253 \\
Ceftriaxone & 129 & 1 & 123 & 253 \\
\hline
\end{tabular}

Out of the 303 isolates, $58.33 \%$ were MDR. $59.09 \% E$. coli isolated and $78.57 .0 \%$ of $S$. aureus isolated were MDR strains. $100 \%$ isolates of C. koseri, K. oxitoca, Enterobacter species and $P$. aeruginosa were MDRstrains.

Of the 98 ESBL screen positive E. coli isolates, 76 isolates were ESBL producers and out of $9 \mathrm{ESBL}$ screen positive $K$. pneumoniae isolates, 7 isolates were ESBL producers by Combination disc method. E. coli and $K$. pneumoniae showed maximum ESBLs production in CAZ/CAZC combination than CTX/ CTXC combination. The in-vitro antibiotic sensitivity pattern of the ESBL E. coli and $K$. pneumoniae isolates to common anti-microbial agents is shown in Table 3 and 4. 
Nepal Journal of Science and Technology Vol. 16, No.1 (2015) 105-112

Table 3. Antibiotic profile in ESBL producing $E$. coli strain

\begin{tabular}{c|l|c|c|c}
\hline S.No. & Name of antibiotics & Sensitive & Resistant & Total \\
\hline 1 & Ampicillin & 4 & 72 & 76 \\
2 & Nalidixic acid & 4 & 72 & 76 \\
3 & Norfloxacin & 9 & 67 & 76 \\
4 & Ciprofloxacin & 3 & 73 & 76 \\
5 & Ofoxacin & 17 & 59 & 76 \\
6 & Nitrofurantoin & 62 & 14 & 76 \\
7 & Gentamicin & 48 & 28 & 76 \\
8 & Amikacin & 75 & 1 & 76 \\
9 & Cotrimoxazole & 20 & 56 & 76 \\
10 & Ceftriaxone & 0 & 76 & 76 \\
11 & Ceftazidime & 0 & 76 & 76 \\
12 & Amoxicillin/Clavulanic acid & 0 & 0 & 76 \\
13 & Imipenem & 76 & & 76 \\
\hline
\end{tabular}

Table 4. Antibiotic profile in ESBL producing K. pneumoniae strain

\begin{tabular}{c|l|c|c|c}
\hline S.No. & Name of antimicrobials & Sensitive & Resistant & Total \\
\hline 1 & Ampicillin & 0 & 7 & 7 \\
2 & Nalidixic acid & 0 & 7 & 7 \\
3 & Norfloxacin & 0 & 7 & 7 \\
4 & Ciprofloxacin & 0 & 7 & 7 \\
5 & Ofoxacin & 0 & 7 & 7 \\
6 & Nitrofurantoin & 0 & 7 & 7 \\
7 & Gentamicin & 0 & 7 & 7 \\
8 & Amikacin & 6 & 1 & 7 \\
9 & Cotrimoxazole & 0 & 7 & 7 \\
10 & Ceftriaxone & 0 & 7 & 7 \\
11 & Ceftazidime & 0 & 7 & 7 \\
12 & Amoxicillin/Clavulanic acid & 0 & 7 & 7 \\
13 & Imipenem & 7 & 0 & 7 \\
\hline
\end{tabular}

Overall, the prevalence of MRSA in the urinary $S$. aureus isolates was found to be $47.61 \%$ using cefoxitin disc. The in-vitro antibiotic sensitivity pattern of the
ESBL E. coli and $K$. pneumoniae isolates to common anti-microbial agents is shown in Table 5.

Table 5. Antibiotic profile in MRSA strain

\begin{tabular}{l|l|c|c|c}
\hline S. No & Antibiotic used & Sensitive & Resistant & Total \\
\hline 1 & Penicillin & 0 & 10 & 10 \\
2 & Cefoxitin & 0 & 10 & 10 \\
3 & Ampicillin & 0 & 10 & 10 \\
4 & Gentamicin & 7 & 3 & 10 \\
5 & Norfloxacin & 3 & 7 & 10 \\
6 & Nitrofurantoin & 10 & 0 & 10 \\
7 & Cotrimoxazole & 7 & 3 & 10 \\
8 & Vancomycin & 10 & 0 & 10 \\
\hline
\end{tabular}

Urinary tract infection is a serious health problem affecting millions of people each year. Urinary tract infections are a common problem in general practice and are usually treated empirically. Empirical treatments should be based on local data regarding common pathogens and their susceptibility to available antibiotics.
The prevalence of UTI in the population was $24.08 \%$. This figure is higher than the $20.2 \%$ rate recorded by Getachew (2010) and 21.8\% rate recorded by Mahmood (2011). The high prevalence may be due to genuine population susceptibility because factors like sexual intercourse, peer group influence, pregnancy, low socio-economic status, are common among Nepali men 
and female. It is lower than prevalence rate of $60 \%$ significant bacteriuria recorded by Kolawole et al. (2009) and $80 \%$ by Durgesh et al. (2012).

Out of 303 cases with significant bacteriuria, $83.49 \%$ were due to gram negative rods and $15.51 \%$ were due to gram positive cocci. In a similar study performed by Mahmood (2011) out of 100 urinary isolates, 85\% constituted Gram negative isolates whereas $15 \%$ Gram Positive Isolates.

The study revealed E. coli and $S$. aureus to be the dominant organisms among other uropathogens. The most common organism isolated in these patients was E. coli $(65.3 \%)$ and this finding is similar to other reports which indicated that a gram negative bacterium, particularly E. coli, was the commonest pathogen isolated in patients with UTI (Mahmood 2011, Kolawole et al. 2009, Devanand \& Saxena 2013, Sasirekha et al. 2010).This was due to the fact that strains of E. coli affecting the urinary tract possess a variety of virulence characteristics that facilitate their intestinal carriage, persistence in vagina and then ascension and invasion of the anatomically normal urinary tract (Annabelle et al. 1999).

Among the common antibiotics used against all Gram negative isolates, Gentamycin was the drug of choice with susceptibility of $75.9 \%$ because 192 out of 253 isolates were found to be susceptible to the drug followed by Nitrofurantoin with a susceptibility of $58.5 \%$. Most of the Gram negative isolates, i.e. $79.5 \%$ (201 out of 253) were resistant to Ampicillin. Nitrofurantoin was found to be the most effective drug against urinary pathogens also in other similar studies by Jha and Yadav (1992).

Among the Gram positive isolates, most of them i.e. $80.9 \%$ were susceptible to Nitrofurantoin followed by Gentamycin with the susceptibility of $68.1 \%$. Nalidixic acid was found to be the least effective as only $14.9 \%$ isolates were sensitive to the drug.

Resistance rates among common uropathogens to many commonly used antimicrobial agents have increased over the years and these resistance rates vary from country to country. Among the $198 \mathrm{E}$. coli isolates, 117 (59.1\%) were isolates were MDR strains. Similarly $100 \%$ of $C$. freundii, $P$. aeruginosa, Klebsiella oxitoca, Enterobacter species were MDR.
ESBLs are paradigmatic as a mechanism of resistance because of the impact they have had on the therapy of infections and the insight they have offered on the relationship between structures and function, in antibiotics and in their determinants of resistance. Of the 198 Uropathogenic E. coli (UPEC) isolates, 98 $(49.5 \%)$ were shown resistance to ceftriaxone and all were subjected for phenotype confirmatory test for ESBL, combination disc method (CLSI 2013). Total 76 isolates were identified as ESBL producers. Poudel (2013) also observed similar results in which 67 out of 222 E. coli isolates were ESBL producers. Out of 20 isolates of K. pneumoniae, 9 isolates showed a resistance to ceftriaxone and 7 were confirmed as ESBL producers.

In our study, resistance to third generation cephalosporins was found to coexist with resistance to two or more antibiotics like ampicillin, nalidixic acid, ciprofloxacin, Ofloxacin, Amoxicillin/Clavulanic acid as also reported by several studies indicating multidrug resistant pattern. Mechanisms of co-resistance are not clear, but one possible mechanism is the cotransmission of ESBL and resistance to other antimicrobials within the same conjugative plasmids (Barber 1948, Levy 1982, Levy 1998, Crofton \& Mitchison 1948, Bradford 2001).

Above $90 \%$ ESBL positive isolates were found resistant to ampicillin, quinolones, fluoroquinolones and amoxicillin/clavulanic acid. High sensitivity of ESBL positive isolates towards imipenem and amikacin advocates the usage of amikacin and carbapenem antibiotics as the therapeutic alternative to beta-lactam antibiotics as indicated in many studies (Bradford 2001, Al-Agamy et al.2006). Nitrofurantoin was considered the most effective of the drugs that can be orally administered; which represent an alternative for oral empiric therapy of uncomplicated UTI and is recommended by the Infectious Disease Society of America (IDSA) (Gupta et al. 2011). Nitrofurantoin in this study demonstrates excellent activity against UPEC isolates i.e. $68 \%$ sensitive; both in complicated and uncomplicated UTIs and also in community acquired and hospital acquired infections. This study showed good co-resistance between nitrofurantoin and ESBL. Moreover, the production of ESBLs has been associated with decreased susceptibility to nitrofurantoin (Carattoli 2009, Laupl and et al. 2008, Rodriguez-Bano \& Navarro 2008). As in UPEC 
infection, rising rates of resistance to antimicrobial drugs limits the choice of drugs that can be used to treat infections with these potent pathogens.

Aztreonam was $100 \%$ resistant in our study, which agrees with those of Sasirekha et al. (2010). Most of the ESBL producing organisms were found to be coresistance to flouroquinolones, aminoglycosides and co-trimoxazole, which correlates with the study done by Denholm and Jabeen (Denholm et al. 2009, Jabeen etal.2005). This was due to the genes encoding ß-lactamases were often located on large plasmids that also encode genes for resistance to others antibiotics, including aminoglycosides, tetracycline, sulfonamides, trimethoprime and chloramphenicol (Perez et al. 2007). We found such associated resistance with fluroquinolones.

In this study we used two combinations with clavulanic acid (CAZ/CAZC and CTX/CTXC) and found that E. coli and $K$. pneumoniae showed maximum ESBLs production in CAZ/CAZC combination, which correlates with other studies (Rahman et al.2004, Thomson et al. 1991).

The overall methicillin-resistant $S$. aureus among $S$. aureus urinary tract infection was found to be $47.61 \%$. The prevalence of MRSA in this study was higher (27.4\%) than the reported prevalence of MRSA among uropathogens by Sashirekha et al. 2010. The high prevalence of MRSA among UTI patients in this study may suggest that MRSA is more prevalent in $S$. aureus.

\section{Conclusion}

Based on our study, it is concluded that UTI affected females more than males. The main organism causing UTI is E. coli followed by S. aureus. Almost all isolates are resistant to commonly prescribed antibiotics. Therefore, antibiotics should only be commenced after performing culture sensitivity test because most of the UTI patients are treated empirically with different antibiotics.

Infection-control practitioners and clinicians need the clinical laboratory to rapidly identify and characterize different types of resistant bacteria specially ESBLs and MRSA efficiently to minimize the spread of these bacteria and help to select more appropriate antibiotics.

\section{Acknowledgements}

We like to express our special thanks of gratitude to Central Department of Microbiology, Tribhuvan University and KIST Medical College Teaching Hospital for the support to complete this research work.

\section{References}

Al-Agamy, M.H., M.E. Ashour and I. Wiegand. 2006. First description of CTXM B-lactamase-producing clinical Escherichia coli isolates from Egypt. Int. J. Antimicrob. Agent. 27: 545-548.

Annabelle, T., M.D. Dytan, A. Jennifer and M.D. Chua. 1999. Surveillance of pathogens 15 and resistance patterns in urinary tract infections. Phil. J. Microbiol. Infect. Dis . 28(1): 11-14.

Barber, M. 1948. Infection by penicillin resistant Staphylococci. Lancet 2: 641-644.

Barrett, F.F., R.F. McGhee and M. Finland. 1968. Methicillinresistant Staphylococcus aureus at Boston city hospital. New England Journal of Medicine 279: 448.

Boscia, J.A. and D. Kaye. 1987. A symptomatic bacteriuria in the elderly. Infect Dis. Clin. North Am.1: 893-905.

Bradford, P.A. 2001. Extended-spectrum B-lactamases in the $21^{\text {st }}$ century: characterization, epidemiology, and detection of this important resistance threat. Clin. Microbiol. Rev.14: 933-951.

Carattoli, A. 2009. Resistance plasmid families in Enterobacteriaceae. Antimicrob Agents Chemother. 53: 2227-2238.

Cheesbrough, M. 1984. Medical Laboratory Manual for Tropical Countries Microbiology. $1^{\mathrm{st}}$ ELBS ed.Cambridge: University Press.

Cheesbrough, M. 2000. District Laboratory Practice in Tropical Countries. Part 2, Cambridge University Press.

CLSI. 2013. Performance Standards for Antimicrobial Susceptibility Testing, Twenty-Third Informational Supplement, CLSI Document M100-S23, Wayne, PA: Clinical and Laboratory Standards Institute.

Collee, J.G., A.G. Fraser, B.P. Marmion and A. Simmons. 1999. Mackie and McCartney Practical Medical Microbiology, $14^{\text {th }}$ edition, Churchill Livingstone, New York, USA.

Crofton, J and D.A. Mitchison. 1948. Streptomycin resistance in pulmonary tuberculosis. Br. Med. J. 2: 1009-1015.

Denholm, J.T., M. Huysmans and D. Spelman. 2009. Community acquisition of ESBL-producing Escherichia coli: a growing concern. Medical Journal Australia 190: 45-46.

Devanand, P and Saxena. 2013. Prevalence and distribution of gram negative bacteria of Enterobacteriaceae causing Urinary tract infections among hospitalized patients. $J$. Acad. Indus. Res. 1(11): 50-53. 
Durgesh, D., Wasnik, P.M. Tumane. 2012. Prevalence and antibacterial susceptibility pattern of Urinary Tract Infection Causing Human Pathogenic Bacteria. Asian Journal of Biomedical and Pharmaceutical Sciences 2 (15): 1-3.

Forbes, B.A., D.F. Sahm and A.S. Weissfeld. 2002. Bailey and Scott's Diagnostic Microbiology, $11^{\text {th }}$ edition. Mosby, Inc. USA.

Fowler, J.E. and M. Mariano. 1990. Immunologic response of the prostate to bacteriuria and bacterial prostates: Antigen specific immunoglobulin in men with bacterial prostatitis. J Urol. 131: 363.

Getachew, T. 2010. Bacterial pathogens implicated in causing urinary tract infection (UTI) and their antimicrobial susceptibility pattern in Ethiopia Revista CENIC. Centro Nacional de Investigaciones Científicas Cuba 41: 1-6.

Gupta, K., T.M. Hooton, K.G. Naber, B. Wullt, R. Colgan, L.G. Miller, G.J. Moran, L.E. Nicolle, R. Raz, A.J. Schaeffer and D.E. Soper. 2011. International Clinical Practice Guidelines for the Treatment of Acute Uncomplicated Cystitis and Pyelonephritis in Women:A 2010 Update by the Infectious Diseases Society of America and the European Society for Microbiology and Infectious Diseases 52: 103-120.

Jabeen. K., A. Zafar, R. Hasan. 2005. Frequency and sensitivity pattern of extended spectrum beta lactamase producing isolates in a tertiary care hospital laboratory of Pakistan. Journal Pakistan Medical Association 55: 436-439.

Jha, V.C. and J.N. Yadav. 1992. Bacterial species isolated from urine of UTI patients and their sensitivity to commonly available antibiotic. J Nep Med Assoc. 30: 222-225.

Kolawole, A.S., O.M. Kolawole, Y.T. Kandaki-Olukemi, S.K. Babatunde, K.A. Durowade and C.F. Kolawole. 2009. Prevalence of urinary tract infections (UTI) among patients attending Dalhatu Araf Specialist Hospital. International Journal of Medicine and Medical Sciences 1: 163-167.

Laupland, K.B., D.L. Church, J. Vidakovich, M. Mucenski and J.D. Pitout.2008. Community-onset extendedspectrum B-lactamase (ESBL) producing Escherichia coli: importance of international travel. J Infect. 57: 441-448.

Lee, J.H. 2003. Methicillin (Oxacillin)-resistant Staphylococcus aureus strains isolated from major food animals and their potential transmission to humans. Appl Environ Microbiol. 69(11): 6489-6494.

Levy, S.B. 1982. Microbial resistance to antibiotics. An evolving and persistent problem. Lancet 2: 83-88.

Levy, S.B. 1998. The challenge of antibiotic resistance. Sci. Am. 278: 46-53.

Mahmood, M.A. 2011. Department of Basic Sciences, College of Dentistry, Baghdad University, Baghdad-Iraq.

Maucky, H.F. 2011. Methicilin resistant Staphylococcusaureus skin, soft tissue infection among HIV infected individuals attending HIV care and treatment clinics in Dar es Salaam city, Tanzania. Muhimbili University of Health and Allied Sciences (MUHAS). 15.

Mshana, S.E., E. Kamugisha, et al. 2009. Prevalence of clindamycin inducible resistance among methicillinresistant Staphylococcus aureus at Bugando Medical Centre, Mwanza, Tanzania. Tanzania Journal of Health Research 11(2): 59-64.

Peirano, G., J.D.D Pitout. 2010. Molecular epidemiology of Escherichia coli producing CTX-M B, Lactamases: worldwide emergence of clone ST131 025:H4. International journal of Antimicrobial Agents 35: 316321.

Perez, F., A. Endimiani, K.M. Hujer and R.A. Bonomo. 2007. The continuing challenge of ESBLs. Current Opinion in Pharmacology 7: 459-469.

Pinho, M.G., H. de Lencastre and A. Tomasz. 2001. An acquired and a native penicillin-binding protein cooperate in building the cell wall of drug-resistant staphylococci. Proc Natl Acad Sci U S A. 98(19): 10886-10891.

Pokhrel, B.M. 2004. A Handbook of Clinical Microbiology. $1^{\text {s }}$ ed. Gorakhnath Desktop and Printing Supports, Kathmandu, Nepal.

Poudel, S. 2013. Status of extended spectrum beta lactamase producing Enterobacteriaceae among bacterial uropathogens. Central Department of Microbiology, TU, Kirtipur, Nepal. 23-55.

Rahman, M.M., J.A. Haque, M.A. Hossain, R. Sultana, F. Islam and S. Islam. 2004. Prevalence of extended spectrum beta lactamase-producing Escherichia coli and Klebsiella pneumoniae in an urban hospital in Dhaka Bangladesh. International journal of antimicrobial agents. 24: 508-510.

Rodriguez-Bano, J., M.D. Navarro. 2008. Extended-spectrum B-lactamases in ambulatory care: a clinical perspective. Clin Microbiol Infect. 14: 104-110.

Sapkota, B., R.K. Bhandari and B.R. Tiwari. 2014. Susceptibility Pattern of Gram Negative Urine Pathogens with Exclusion of Escherichia coli to Quinolone/ Fluoroquinolone Antibiotics. Nepal Journal of Science and Technology. 15(2): 77-84.

Sasirekha, B., R. Manasa, P. Ramya and R. Sneha. 2010. Frequency and antimicrobial sensitivity pattern of extended spectrum $\beta$-Lactamases producing E. coli and Klebsiella pneumoniae isolated in a tertiary care hospital. Al Ameen journal Medical science. 3: 265-271.

Thomson, K.S., C.C. Sanders and J.A. Washington. 1991. Highlevel resistance to cefotaxime and ceftazidime in Klebsiella pneumoniae isolates from C level and, Ohio. Antimicrob Agents Chemother. 35: 1001-1003.

Weese, J.S., M. Archambault, B.M. Willey, P. Hearn, B.N. Kreiswirth, B. Said-Salim, A. McGeer, Y. Likhoshvay, J.F. Prescott and D.E. Low. 2005. Methicillin-resistant Staphylococcus aureus in horses and horse personnel, 2000-2002. Emerg Infect Dis. 11(3): 430-435. 
Nepal Journal of Science and Technology Vol. 16, No.1 (2015) 105-112 